\title{
Representation of Functional Data in Neural Networks *
}

\author{
Fabrice Rossi $^{\mathrm{a}, \mathrm{b}, *}$, Nicolas Delannay ${ }^{\mathrm{c}, 1}$, Brieuc Conan-Guez ${ }^{\mathrm{a}}$ \\ and Michel Verleysen ${ }^{\mathrm{c}, 2}$

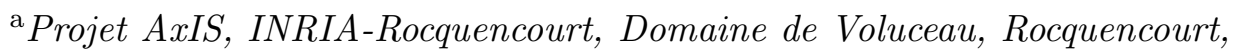 \\ B.P. 105, 78153 Le Chesnay Cedex, France \\ ${ }^{\mathrm{b}}$ CEREMADE, UMR CNRS 7534, Université Paris-IX Dauphine, \\ Place du Maréchal de Lattre de Tassigny, 75016 Paris, France \\ ${ }^{\mathrm{c}}$ Université catholique de Louvain (UCL) DICE - Machine Learning Group \\ Place du levant 3, 1348 Louvain-la-Neuve, Belgium
}

\footnotetext{
ऋ Published in Neurocomputing (Volume 64, pages 183-210). DOI: http://dx. doi.org/10.1016/j.neucom.2004.11.012

* Corresponding author:

Fabrice Rossi

Projet AxIS

INRIA Rocquencourt

Domaine de Voluceau, Rocquencourt, B.P. 105

78153 LE CHESNAY CEDEX - FRANCE

Tel: (33) 139635445

Fax: (33) 139635892

Email addresses: Fabrice.Rossi@inria.fr (Fabrice Rossi), delannay@dice.ucl.ac.be (Nicolas Delannay), Brieuc.Conan-Guez@inria.fr (Brieuc Conan-Guez), verleysen@dice.ucl.ac.be (Michel Verleysen).

1 Nicolas Delannay is Research Fellow of the Belgian National Fund for Scientific Research.

2 Michel Verleysen is Senior Research Associate of the Belgian National Fund for Scientific Research.
}

Preprint submitted to Elsevier Science 


\title{
Representation of Functional Data in Neural Networks
}

\begin{abstract}
Functional Data Analysis (FDA) is an extension of traditional data analysis to functional data, for example spectra, temporal series, spatio-temporal images, gesture recognition data, etc. Functional data are rarely known in practice; usually a regular or irregular sampling is known. For this reason, some processing is needed in order to benefit from the smooth character of functional data in the analysis methods. This paper shows how to extend the Radial-Basis Function Networks (RBFN) and Multi-Layer Perceptron (MLP) models to functional data inputs, in particular when the latter are known through lists of input-output pairs. Various possibilities for functional processing are discussed, including the projection on smooth bases, Functional Principal Component Analysis, functional centering and reduction, and the use of differential operators. It is shown how to incorporate these functional processing into the RBFN and MLP models. The functional approach is illustrated on a benchmark of spectrometric data analysis.
\end{abstract}

Key words: Functional data analysis, smooth data, projection on smooth bases, irregular sampling, missing data 


\section{Introduction}

Many modern measurement devices are able to produce high-resolution data resulting in high-dimensional input vectors. A promising way to handle this type of data is to make explicit use of their internal structure. Indeed, highresolution data can frequently be identified as discretized functions: this is the case for time series (in the time domain as well as in the frequency domain), spectrometric data, weather data (in which we can have both time and location dependencies), etc. Functional Data Analysis (FDA, see [21]) is an extension of traditional data analysis methods to this kind of data. In FDA, each individual is characterized by one or more real-valued functions, rather than by a vector of $\mathbb{R}^{p}$. Function estimates are constructed from high-dimensional observation vectors and data analysis (in a broad sense) is carried out on those estimates.

As it is not possible to directly manipulate arbitrary functions, a computerfriendly representation of functional data must be used: this is obtained through a basis expansion in the functional space, for instance with a B-spline approximation. This way of proceeding has numerous advantages over a basic multivariate analysis of high-dimensional data. Indeed the choice of a fixed basis allows to introduce prior knowledge: for instance a Fourier basis can be used to model periodic functions such as daily temperature observations in a fixed location. A fixed basis also allows to deal with irregularly sampled functions and with missing data. A side effect of the representation is that it can be used to smooth the data either individually or globally (see [3] ). Another interesting point is that most FDA methods can work directly on the numerical coefficients of the basis expansion, leading to far less computational burden. An additional advantage of dealing with functions is the possibility of using functional preprocessing such as derivation, integration, etc.

FDA is based on the fact that the first step performed by many data analysis methods consists in simple operations on the data: distance, scalar product and linear combination calculations. Those operations can be defined in a satisfactory way in arbitrary Hilbert spaces that include functional spaces (such as $L^{2}$ ). This means that many data analysis methods can be extended to work directly with functional inputs. There are of course some theoretical difficulties induced by the infinite dimension of the considered spaces. Nevertheless, traditional data analysis methods have been successfully adapted to functional data, both on theoretical and practical point of views. We refer to [21] for a comprehensive introduction to those methods, especially to functional principal component analysis and functional linear models. For regression and discrimination problems, recent developments of FDA include nonlinear models such as multilayer perceptrons [26, 25], semi-parametric [15] and non-parametric models [13, 14]. 
In this paper, we extend the results from [11, 23], and introduce two nonlinear neural models for functional data. They are adaptation of classical neural models, the Radial-Basis Function Network (RBFN) and the Multi-Layer Perceptron (MLP), to functional inputs. In particular, we show how to implement these functional models in practical situations, when the functional data are known through a list of numerical samples (regularly or irregularly sampled input-output pairs). In section 2 we first show that many data analysis algorithms can be defined on arbitrary Hilbert spaces, that include the $L^{2}$ functional space with its elementary operations. We illustrate this general construction with the proposed models, using distances in $L^{2}$ for the RBFN and inner products for the MLP. Section 3 introduces the general FDA method that uses function representation to actually implement theoretical models defined in $L^{2}$. We show that the MLP and RBFN models can be implemented on preprocessed coordinates of the functional data, providing a way to easily introduce functional data processing in classical neural software. We also introduce in this section natural preprocessings that are available for functional data, such as functional principal component analysis, derivation, etc. In section 4 we report simulation results on a real-world benchmark, a spectrometric problem in which the percentage of fat contained in a meat sample must be predicted based on its near-infrared spectrum. We show that functional preprocessing greatly improves the performances of the RBFN and gives very good performances with a simple MLP. In section 5, we build a semi-artificial dataset, introducing randomly placed holes in the spectrum data. This simulates irregular sampling in its simplest form (missing data). We show that the functional reconstruction allows to maintain excellent predictions whereas classical data imputation techniques are not able to reconstruct the missing information.

\section{Working directly in functional spaces}

\subsection{Introduction}

In this paper, we focus on regular functions, i.e. on square integrable functions from $V$, a compact subset of $\mathbb{R}^{p}$, to $\mathbb{R}$. We denote $L^{2}(V)$ the vector space of those functions. A $L^{2}(V)$ space equipped with its natural inner product $\langle f, g\rangle=\int_{V} f(x) g(x) \mathrm{d} x$, is a Hilbert vector space. In the present section, we will avoid using specific aspects of $L^{2}(V)$. We will rather illustrate how elementary operations available in a Hilbert space as linear combinations, inner product, norm and distance calculations are sufficient to implement many data analysis algorithms, at least on a theoretical point of view.

In this section, $H$ denotes an arbitrary Hilbert space. When $u$ and $v$ are 
arbitrary elements of $H,\langle u, v\rangle$ denotes their inner product, and $\|u\|=\sqrt{\langle u, u\rangle}$ the norm of $u$.

\subsection{Data analysis in a Hilbert space}

Even if data analysis algorithms have been defined for traditional multivariate observations, they seldom use explicitely the finite dimensional character of the input spaces. The most obvious cases are distance-based algorithms such as the $k$-means method.

Indeed, the $k$-means algorithm clusters input data by alternating between two phases: an affectation phase and a representation phase. The goal is to obtain representative clusters; each of them is defined by a prototype that belongs to the input space. Given the prototypes, the affectation phase puts input vector $x$ in the cluster defined by the prototype closest to $x$ : obviously we only need to calculate distances between points in the input space to perform this affectation operation. The representation phase consists in updating the prototypes given by the results of the affectation phase. For a given cluster, the new prototype is defined as the center of gravity of the input vectors associated to the considered cluster. The new prototype is therefore a linear combination of input vectors. Hence $k$-means can be defined for any normed vectorial input space (an inner product is not even needed); this obviously includes functional spaces. The $k$-means method has been adapted to $L^{2}$ spaces in [2] in which the consistency of the algorithm is proved (see also [17] for a EM-like version of a functional clustering algorithm).

More sophisticated clustering methods such as the Self-Organizing Map (SOM, [18]) are also based on elementary operations (distance and linear combination calculations). They can therefore be applied to functional input spaces (see [24] for the SOM applied to functional data).

Regression models can also be constructed when the explanatory variable belongs to arbitrary normed vector spaces. Let us consider for instance the linear regression: the goal is to model a random variable $Y$ (the target variable with values in $\mathbb{R}$ ) as a linear function of a random vector $X$ (the input variable), i.e. $E(Y \mid X)=l(X)$. If $X$ has values in $\mathbb{R}^{p}$, an explicit numerical representation of the linear function can be written such as $l(X)=\sum_{i=1}^{p} \alpha_{i} X_{i}$, where $X_{i}$ is the $i$-th coordinate of $X$. More generally if $X$ has values in an arbitrary normed vector space $M$, it is still possible to model $Y$ by $E(Y \mid X)=l(X)$ by requesting $l$ to belong to $M^{*}$, the topological dual of $M$, i.e. the set of continuous linear functions from $M$ to $\mathbb{R}$. In the particular case of a Hilbert space $H$, the identification of $H$ with its dual $H^{*}$ is used to obtain a simpler formulation. More precisely, any continuous linear form $l$ on $H$ can be represented through 
an element $v \in H$ such that $l(u)=\langle u, v\rangle$. We have therefore $E(Y \mid X)=\langle X, v\rangle$ for a well chosen $v \in H$.

Of course, the linear model is a very limited regression model. Non-parametric models are a possible solution to overcome those limitations: they have been extended to functional data in [13, 14]. Semi-parametric models have also been adapted to functional inputs in [15]. We propose in this paper to build neural network based nonlinear regression models. In the following sections, we show how to define Radial-Basis Function Networks (RBFNs) and MultiLayer Perceptrons (MLPs) with functional inputs.

\subsection{Radial-Basis Function Networks}

Radial-Basis Function Networks (RBFN) are popular nonlinear models that have several advantages over other nonlinear regression paradigms. Besides their simplicity, their intuitive formulation and their local approximation abilities, their most important advantage is probably the ability of various learning procedures to avoid the local minima issue, for example when the parameters of the model are the solution of a linear problem.

The first operation performed by RBFN models on the input data is based on the notion of distances. The following of this section shows how this notion can be inserted in a functional data context and more generally in any metric space such as a Hilbert space.

The output of a RBF network is expressed by

$$
y=\sum_{i=1}^{p} \alpha_{i} \varphi_{i}\left(d_{i}\left(x, c_{i}\right)\right)
$$

where $x$ is the input of the network, $y$ its scalar output, $\varphi_{i}(\cdot)$ are radial-basis functions from $\mathbb{R}$ to $\mathbb{R}, c_{i}$ are centers chosen in the input data space of $x$, $d_{i}(\cdot, \cdot)$ are associated distances and $\alpha_{i}$ are weighting coefficients. We see that the predicted output is expressed as a weighted sum of basis functions with radial shape (each basis function has a radial symmetry around a center). This property is very general; any distance measure between the input $x$ and the centers $c_{i}$ could be used. Most frequently the RBF are Gaussian $\varphi_{i}(r)=$ $\exp \left(-r^{2}\right)$.

Equation 1 easily generalizes to any metric space by replacing all distances $d_{i}$ by the distance used to define the space. In the particular case of a functional space, distance $d_{i}\left(x, c_{i}\right)$ between vectors is simply replaced by a distance $d_{i}\left(g(\cdot), c_{i}(\cdot)\right)$ between the functional input $g(\cdot)$ and the functional centers $c_{i}(\cdot)$. 
Some RBF (e.g. Gaussian functions) can define the positive definite kernel of a Reproducing Kernel Hilbert Space (RKHS) $k\left(x, x^{\prime}\right)=\varphi\left(d\left(x, x^{\prime}\right)\right)$. Regularization networks [16] and Support Vector Machines (SVM) result from a learning theory within this RKHS context [31, 12]. Therefore the following discussion straightforwardly applies to these models too.

RBF networks have the universal approximation property [20]. In practice, when a finite number of observed data is available, the way the distance is defined plays a crucial role on the generalization performances of the network. Dealing with functional data, the kind of distance to be used must be specified. For example, considering functions from $L^{2}(V)$, the Euclidean distance in this space could be chosen:

$$
d_{i}\left(g(\cdot), c_{i}(\cdot)\right)=\left(\int_{V}\left(g(x)-c_{i}(x)\right)^{2} d x\right)^{1 / 2} .
$$

While this choice might reveal adequate in some situations, the Euclidean distance is in fact quite restrictive. In the vectorial case, one could use a weighted distance (for example the Mahalanobis one) instead of the Euclidean distance; generalizing to functional spaces, a weighted version of the Euclidean distance could be used to characterize the measure of locality around each center:

$$
d_{i}\left(g(\cdot), c_{i}(\cdot)\right)=\left(\int_{V} \int_{V}\left(g\left(x^{\prime}\right)-c_{i}\left(x^{\prime}\right)\right) w_{i}\left(x^{\prime}, x\right)\left(g(x)-c_{i}(x)\right) d x d x^{\prime}\right)^{1 / 2},
$$

where $w_{i}\left(x^{\prime}, x\right)$ is a positive definite bivariate function over $V \times V$. While this last definition is very general, there is unfortunately no simple way to choose the weighting function $w_{i}\left(x^{\prime}, x\right)$. It seems reasonable to look for a weighting function that shows approximately the same complexity as the input data (the complexity of a function being often measured through its second derivative). Using a smooth weighting function leads to the so-called functional regularization. In other words, working with functional data necessitates a functional regularization of the parameters defining the distance measure.

Another possibility resulting from the use of functional data is that differential operators can be applied. This could reveal interesting for example when the shape of the functional inputs is known to be more important than their absolute levels (or means); see section 4.3 for an application example. In the framework of RBF networks, differential operators can also be included in the distance function, which becomes a semi-metric:

$$
d_{i}\left(g(\cdot), c_{i}(\cdot)\right)=\left(\int_{V}\left(D g(x)-c_{i}(x)\right)^{2} d x\right)^{1 / 2},
$$

where $D(\cdot)$ is a differential operator (for example the first or second derivative, as used in sections 4 and 5). 


\subsection{Multi-layer Perceptrons}

A multilayer perceptron (MLP) consists in neurons that perform very simple calculations. Given an input $x \in \mathbb{R}^{p}$, the output of a neuron is

$$
T\left(\beta_{0}+\sum_{i=1}^{p} \beta_{i} x_{i}\right),
$$

where $x_{i}$ is the $i$-th coordinate of $x, T$ is a nonlinear activation function from $\mathbb{R}$ to $\mathbb{R}$, and $\beta_{0}, \ldots, \beta_{p}$ are numerical parameters (the weights of the neuron).

As for the linear model considered previously, this calculation can be generalized to any normed vector space $M$ (see [27, 28, 29]). If $l$ is a linear form in $M^{*}$, it can be used to define a neuron with an input in $M$ and whose output is given by $T\left(\beta_{0}+l(x)\right)$ for $x \in M$. The linear form replaces parameters $\beta_{1}, \ldots, \beta_{p}$. Obviously, the case $M=\mathbb{R}^{p}$ corresponds exactly to the traditional numerical neuron.

In a Hilbert space $H$, linear forms are represented by inner products and define a generalized neuron with an input in $H$ : given an input vector $u$, the neuron output is $T\left(\beta_{0}+\langle u, v\rangle\right)$. The "connection weights" of the neuron are the numerical value $\beta_{0}$ and the vector $v \in H$. In the particular case of $H=L^{2}(V)$, given an input function $g$, the neuron output is $T\left(\beta_{0}+\int_{V} g(x) w(x) \mathrm{d} x\right)$. The neuron is called a functional neuron and $w$ is its weight function.

As the output of a generalized neuron is a numerical value, we need such neurons only in the first layer of the MLP. Indeed, the second layer uses only outputs from the first layer which are real numbers and therefore consists in numerical neurons.

We have presented in [26] and [22] some theoretical properties of MLPs constructed by combining a layer of generalized neurons with inputs in $L^{2}(V)$ and at least one layer of numerical neurons. We use specific properties of $L^{2}(V)$ that allow to restrict the set of "connection weights": rather than working with arbitrary weight functions in $L^{2}(V)$, we use weight functions that can be exactly calculated by a traditional MLP or by any other sufficiently powerful function approximation method. An important result is that MLPs with functional inputs are universal approximators as long as they use sufficiently regular activation functions, exactly as numerical MLPs: given a continuous function $G$ from $K$ a compact subset of $L^{2}(V)$ to $\mathbb{R}$ and $\epsilon>0$ an arbitrary positive real number, there is an one-hidden layer perceptron that calculates a function $H$ such that $|G(g)-H(g)|<\epsilon$ for all $g \in K$. This MLP uses functional neurons in its first (hidden) layer and one numerical neuron (with the identity activation function) in its output layer. We have also shown in [7] that even if the MLP is implemented through a function representation 
(as it will be described in the following section), the universal approximation property is still valid.

\section{Function representation}

\subsection{Functional data in practice}

The previous section shows that it is possible to define many data analysis algorithms for arbitrary Hilbert spaces. However, the proposed solutions are purely theoretical; it is in general impossible to manipulate arbitrary functions from $L^{2}(V)$ on a computer. Moreover, functional data coming from sensors, measurements or collected in other ways do not consist in mathematical functions. On the contrary, as stated in the introduction, observations are discretized functions: each of them is a list of input/output pairs. These lists may include missing data or more generally show irregular sampling: the sets of inputs for each observation do not necessarily coincide.

More precisely, let us assume that we observe $n$ functions such that function $i$ is given by the $\left(x_{j}^{i}, y_{j}^{i}\right)_{1 \leq j \leq m^{i}}$ list of $m^{i}$ pairs, with $x_{j}^{i} \in V$ and $y_{j}^{i} \in \mathbb{R}$. FDA main assumption is that there is a regular function $g^{i}$ (in $L^{2}(V)$ ) such that $y_{j}^{i}=g^{i}\left(x_{j}^{i}\right)+\epsilon_{j}^{i}$, where $\epsilon_{j}^{i}$ is an observation noise. In this model, both the number of observations $m^{i}$ and the $\left(x_{j}^{i}\right)_{1 \leq j \leq m^{i}}$ can depend on $i$.

The $g^{i}$ functions are not known. This prohibits the straightforward application of the models developed in the previous section. Even with known functions, calculating elementary operations, such as integrals, is difficult. Nevertheless the rationale of FDA is to implement theoretical models on those functions. A possible solution, quite common in FDA methods, is to construct an approximation of the $\left(g^{i}\right)_{1 \leq i \leq n}$ and then to work on these approximations. One way to build them is to project the original $g^{i}$ on a known subspace.

\subsection{Representation on a subspace}

FDA introduces some specific needs that have to be taken into account to choose a representation subspace. A first need is that the representation must be computed for every input list; this computation should therefore be as fast as possible. A second constraint is that the operations performed on the reconstructed functions must approximate as exactly as possible the corresponding operations on the original $g^{i}$ functions. 


\subsubsection{An approximate projection}

A simple and efficient solution is provided by a projection approach which makes use of the $L^{2}(V)$ Hilbert structure. A set of $q$ linearly independent functions from $L^{2}(V),\left(\phi_{k}\right)_{1 \leq k \leq q}$ is chosen. Rather than working on $L^{2}(V)$, we restrict ourselves to $\mathcal{A}=\operatorname{span}\left(\phi_{1}, \ldots, \phi_{q}\right)$ and use $\left(\phi_{k}\right)_{1 \leq k \leq q}$ as a basis for this subspace. Each function $u \in \mathcal{A}$ is represented by its $\alpha_{k}(u)$ coordinates, such that $u=\sum_{k=1}^{q} \alpha_{k}(u) \phi_{k}$.

Given a list $\left(x_{j}^{i}, y_{j}^{i}\right)_{1 \leq j \leq m^{i}}$, the underlying function $g^{i}$ is then approximated by a function $\tilde{g}^{i}$ in $\mathcal{A}$. The best approximation in the functional sense would be to choose $\tilde{g}^{i}$ as the orthogonal projection of $g^{i}$ on $\mathcal{A}$. Obviously, such projection cannot be calculated exactly as we do not know $g^{i}$. Therefore, $\tilde{g}^{i}$ is defined by its numerical coefficients $\left(\alpha_{k}\left(\tilde{g}^{i}\right)\right)_{1 \leq k \leq q}$ chosen to minimize:

$$
\sum_{j=1}^{m^{i}}\left(y_{j}^{i}-\sum_{k=1}^{q} \alpha_{k}\left(\tilde{g}^{i}\right) \phi_{k}\left(x_{j}^{i}\right)\right)^{2} .
$$

This minimization is a standard quadratic optimization problem that can be conducted very efficiently with cost at most $O\left(m^{i} q^{2}\right)$ (see [21] chapter 3 for instance). Moreover, some specific functional bases such as B-splines lead to even faster algorithms with cost $O\left(m^{i} q\right)$ in some situations.

In the next subsection, it is shown that this representation approach also allows to transform the functional operations on $\tilde{g}^{i}$ into calculations on the $\alpha_{k}\left(\tilde{g}^{i}\right)$ coordinates.

\subsubsection{Working with the coefficients}

As $\mathcal{A}$ is a finite-dimensional space, it is possible to work with the coordinates $\alpha_{k}\left(\tilde{g}^{i}\right)$ instead of working directly on the $\tilde{g}^{i}$ functions. Nevertheless, it is shown in the following that additional precautions must be taken if the basis functions $\left(\phi_{k}\right)_{1 \leq k \leq q}$ are not orthonormal.

Once each functional input data is transformed into a vector in $\mathbb{R}^{q}$ that corresponds to its coordinates in $\mathcal{A}$, traditional data analysis algorithms can be used directly on those vectors. However, while this simple approach can give good results in some situations, it introduces an unwanted distortion in the input function representation.

The case of linear operations does not introduce any problem. Indeed a linear combination of functions may be expressed as a linear combination of their coordinate vectors: if $u$ and $v$ are functions in $\mathcal{A}$ and $\lambda$ and $\mu$ are real numbers, then $\alpha_{k}(\lambda u+\mu v)=\lambda \alpha_{k}(u)+\mu \alpha_{k}(v)$ for all $k$. 
On the contrary, inner products, and therefore distances, are a source of problems. Indeed the inner product between two functions in $\mathcal{A}$ can also be defined in terms of their coordinate vectors. The inner product between $u$ and $v$ in $\mathcal{A}$ is given by:

$$
\langle u, v\rangle=\sum_{k=1}^{q} \sum_{l=1}^{q} \alpha_{k}(u) \alpha_{l}(v)\left\langle\phi_{k}, \phi_{l}\right\rangle
$$

If we denote $\alpha(u)=\left(\alpha_{1}(u), \ldots, \alpha_{q}(u)\right)^{T}$, where ${ }^{T}$ is the transposition operator, the inner product becomes:

$$
\langle u, v\rangle=\alpha(u)^{T} \Phi \alpha(v)
$$

where $\Phi$ is the matrix defined by $\Phi_{k l}=\left\langle\phi_{k}, \phi_{l}\right\rangle$ and independent from $u$ and $v$.

This last formula shows that a distortion corresponding to $\Phi$ results from the transition between the inner product $\langle u, v\rangle$ in $\mathcal{A}$ and the canonical inner product in $\mathbb{R}^{q}$. The norm of the difference between functions of course shows the same behavior: in general, $\|\alpha(u)-\alpha(v)\|^{2}$ is different from $\|u-v\|^{2}$, as the latter is given by $(\alpha(u)-\alpha(v))^{T} \Phi(\alpha(u)-\alpha(v))$, while the former is simply $(\alpha(u)-\alpha(v))^{T}(\alpha(u)-\alpha(v))$. If the set of functions $\left(\phi_{k}\right)_{1 \leq k \leq q}$ is not orthonormal, $\Phi$ is different from the identity matrix and the inner product that should be used in $\mathbb{R}^{q}$ is not the canonical one. Unfortunately, some very useful sets of functions, such the B-splines (see section 3.3.1), are not orthonormal.

A simple solution to this problem is to use the Choleski decomposition of $\Phi$, i.e. a square matrix $U$ such that $\Phi=U^{T} U$. The coordinate vectors can then be scaled by matrix $U$ to give $\beta(u)=U \alpha(u)$. Obviously, we have:

$$
\beta(\lambda u+\mu v)=\lambda \beta(u)+\mu \beta(v)
$$

and

$$
\beta(u)^{T} \beta(v)=\sum_{k=1}^{q} \beta_{k}(u) \beta_{k}(v)=\langle u, v\rangle .
$$

This means that performing elementary operations in $\mathbb{R}^{q}$ (with its canonical inner product) on the coordinates $\beta(u)$ is exactly equivalent to performing the same operations in the inner product space $\mathcal{A}$.

Working with the coordinate vectors $\beta\left(\tilde{g}^{i}\right)$ is thus strictly equivalent to working directly on the $\tilde{g}^{i}$ functions, and equivalent to working with $g^{i}$ under the approximation resulting from equation 6 . A nice consequence of this property is that functional models can be implemented as a preprocessing phase before any classical data analysis software: the preprocessing consists in choosing the projection space (see the following section) and in calculating the coordinate vectors $\beta\left(\tilde{g}^{i}\right)$. Then, those vectors can be submitted to a RBFN or a MLP exactly as classical multivariate data. Optionally, some additional functional 
preprocessing can be implemented before the final transformation (see section 3.5). In general, the calculation of $\Phi$ does not introduce any additional problems as the basis functions are under the practitioner control. With an orthonormal basis such as Fourier series, $\Phi$ is the identity matrix. For other bases, quadrature methods or Monte-Carlo methods can be used to calculate an arbitrarily accurate approximation of $\Phi$.

\subsection{Choosing the projection space}

As the underlying functions are reconstructed according to their approximation in the projection space, the choice of this space has an important impact on the data analysis. The projection space must for instance provide a good approximation of arbitrary functions in $L^{2}(V)$ as there is no a priori reason to restrict the functions to a subspace of $L^{2}(V)$.

\subsubsection{Basis}

Good candidate bases are provided by Hilbert bases of $L^{2}(V)$. Given such a basis $\left(\phi_{k}\right)_{1 \leq k}$, truncation allows to define finite-dimensional subspaces: $\mathcal{A}_{q}=\operatorname{span}\left(\phi_{1}, \ldots, \phi_{q}\right)$. An interesting theoretical property of Hilbert bases is that functions from $L^{2}(V)$ can be approximated more and more accurately by increasing the number $q$ of basis functions. In practice, when the Hilbert basis is fixed, a leave-one-out technique allows to choose $q$ directly from the data (see section 3.3.2). An example of Hilbert basis is given by the Fourier series for $L^{2}([a, b])$, where $[a, b]$ is an interval in $\mathbb{R}$.

Another interesting solution is provided by B-splines. Let us assume that $V=[a, b]$ and let $\pi=\left(t_{0}, t_{1}, \ldots, t_{l+1}\right)$ be a sequence such that $t_{0}=a, t_{l+1}=b$ and $t_{k}<t_{k+1}$ for all $k$. With $\nu$ a positive integer, we denote $\mathcal{S}_{\pi}^{\nu}$ the subset of $L^{2}([a, b])$ defined as follows: a function $f \in L^{2}([a, b])$ belongs to $\mathcal{S}_{\pi}^{\nu}$ if $f$ is $C^{\nu-2}$ on $[a, b]$ and if $f$ is a polynomial of degree $\nu-1$ on each sub-interval $\left[t_{k-1}, t_{k}\right]$ for $1 \leq k \leq l+1$. $\mathcal{S}_{\pi}^{\nu}$ is the set of splines of order $\nu$ on $\pi$. Elements of $\pi$ are the knots of the splines. Splines have interesting properties (see for example [10]). For instance, they can approximate arbitrarily well functions in $L^{2}([a, b])$, provided enough knots are used (i.e. $l$ is large enough). Moreover, $\mathcal{S}_{\pi}^{\nu}$ has a basis which is made of $l+\nu$ functions called the B-splines of order $\nu$ on $\pi$ and denoted $B_{k, \pi}^{\nu}$ for $1 \leq k \leq l+\nu$. B-splines are easy to calculate, have local support and have very good numerical properties: finding coordinates of projected functions with equation 6 is both fast and accurate.

The choice of B-splines as basis functions leads to $\mathcal{A}=$ $\operatorname{span}\left(B_{1, \pi}^{\nu}, \ldots, B_{l+v, \pi}^{\nu}\right)=\mathcal{S}_{\pi}^{\nu}$. As for truncated Hilbert bases, $l$ can be automatically chosen by a leave-one-out method. The choice of $\nu$ is more 
complex as it corresponds to a regularity assumption. The most common choice is $\nu=4$, which corresponds to $C^{2}$ functions. In some situations, it is interesting to work with derivatives of the original functional data, in which case higher values of $\nu$ will be more adapted. The last point to address is the choice of $\pi$. While expert prior knowledge can justify irregular positioning of the knots (for instance more knots in rough parts of the studied functions), in general regular knot positions are used, i.e. $t_{k}=a+k \frac{b-a}{l+1}$.

Of course, this section only presented some of the possible choices for the basis. We refer the interested reader to [21], especially to chapters 3, 4 and 15, for a more detailed discussion on function representations.

\subsubsection{Leave-one-out}

While expert knowledge or practical considerations can help to choose the basis among several possibilities such as B-splines or truncated Hilbert bases, the ideal projection space cannot in general be fixed a priori. A simple solution is to rely on leave-one-out to compare different function approximation models.

Let us consider the situation in which we have to compare two candidate projection sub-spaces, $\mathcal{A}=\operatorname{span}\left(\phi_{1}, \ldots, \phi_{q}\right)$ and $\mathcal{B}=\operatorname{span}\left(\psi_{1}, \ldots, \psi_{l}\right)$. Let us consider for now only one function $g$ given by the list $\left(x_{j}, y_{j}\right)_{1 \leq j \leq m}$. We denote

$$
\tilde{g}\left(x_{j}, \mathcal{A}\right)=\sum_{k=1}^{q} \alpha_{k} \phi_{k}\left(x_{j}\right),
$$

where $\alpha_{k}$ is the $k$-th coordinate of the optimal projection of $g$ in $\mathcal{A}$ determined by minimizing equation 6 . Similarly, we denote

$$
\tilde{g}\left(x_{j}, \mathcal{B}\right)=\sum_{k=1}^{l} \gamma_{k} \psi_{k}\left(x_{j}\right),
$$

where $\gamma_{k}$ is the $k$-th coordinate of the optimal projection of $g$ in $\mathcal{B}$ determined by minimizing equation 6 adapted to the $\left(\psi_{k}\right)_{1 \leq k \leq l}$ basis. Comparing the reconstruction errors given by equation 6 is not relevant, as it leads to overfitting. Instead, a leave-one-out estimate of the reconstruction errors of both models is preferred. More precisely, the $\alpha_{k}^{(-p)}$ are defined as the optimal coefficients found when one observation is removed from the list that defines the considered functional data. The $\alpha_{k}^{(-p)}$ thus minimize

$$
\sum_{j=1, j \neq p}^{m}\left(y_{j}-\sum_{k=1}^{q} \alpha_{k}^{(-p)} \phi_{k}\left(x_{j}\right)\right)^{2} .
$$

The $\beta_{k}^{(-p)}$ coefficients are defined in a similar way on the $\mathcal{B}$ subspace, using the $\psi_{k}$ basis instead of the $\phi_{k}$ one. The leave-one-out score associated to $\mathcal{A}$ is 
then given by:

$$
\operatorname{LOO}(g, \mathcal{A})=\frac{1}{m} \sum_{i=1}^{m}\left(y_{i}-\sum_{k=1}^{q} \alpha_{k}^{(-i)} \phi_{k}\left(x_{i}\right)\right)^{2},
$$

and similarly for $\mathcal{B}$ :

$$
L O O(g, \mathcal{B})=\frac{1}{m} \sum_{i=1}^{m}\left(y_{i}-\sum_{k=1}^{l} \gamma_{k}^{(-i)} \psi_{k}\left(x_{i}\right)\right)^{2} .
$$

In our functional data context, we do not have only one function $g$ given by the list $\left(x_{j}, y_{j}\right)_{1 \leq j \leq m}$, but a set of $g^{i}$ functions known through $\left(x_{j}^{i}, y_{j}^{i}\right)_{1 \leq j \leq m^{i}}$. In that case, in order to choose between the $\mathcal{A}$ and $\mathcal{B}$ projection subspaces, the total cross-validation scores that are obtained by summing the leave-one-out scores obtained on each function $g^{i}$ must be compared.

In general, leave-one-out is a very computationally-intensive operation. Fortunately, this is not the case with linear representations, as the expansions on $\mathcal{A}$ and $\mathcal{B}$ chosen in the previous section. Indeed, as the optimization problem of equation 6 is quadratic, there is a matrix $S(\mathcal{A})$ (resp. $S(\mathcal{B})$ ) such that $\tilde{g}(x, \mathcal{A})=S(\mathcal{A}) y(\operatorname{resp} . \tilde{g}(x, \mathcal{B})=S(\mathcal{B}) y)$, where $\tilde{g}(x, \mathcal{A})=$ $\left(\tilde{g}\left(x_{1}, \mathcal{A}\right), \ldots, \tilde{g}\left(x_{m}, \mathcal{A}\right)\right)^{T}$ and $y=\left(y_{1}, \ldots, y_{m}\right)^{T}$. Then, we have (see [21] chapter 10 for instance):

$$
\operatorname{LOO}(g, \mathcal{A})=\frac{1}{m} \sum_{i=1}^{m}\left(\frac{y_{i}-\tilde{g}\left(x_{i}, \mathcal{A}\right)}{1-S(\mathcal{A})_{i i}}\right)^{2}
$$

A similar equation is satisfied by $L O O(g, \mathcal{B})$. In general, the calculation of $S$ is much more efficient than the direct calculation of the cross-validation score.

\subsection{Functional principal component analysis}

Even if the representation on a subspace allows to take into account irregular sampling and very high original input dimensions, it happens frequently in practice that a rather high number of basis functions has to be used to keep a good accuracy for the input function reconstructions. Unfortunately, many data analysis methods suffer from the curse of dimensionality and are therefore not really adapted to a high number of input features. In traditional numerical settings, a simple solution consists in working on a few principal components.

Principal Component Analysis (PCA) was one of the first data analysis methods adapted to functional data (see [8, 9, 4]). On a theoretical point of view functional PCA consists, as traditional PCA, in finding an optimal subspace 
representation. Given $n$ functions $g^{1}, \ldots, g^{n}$ in $L^{2}(V)$, their $q$ principal functions are defined as $q$ orthonormal functions $\xi_{1}, \ldots, \xi_{q}$ such that the following distortion is minimized:

$$
\sum_{i=1}^{n}\left\|g^{i}-\sum_{k=1}^{q}\left\langle g^{i}, \xi_{k}\right\rangle \xi_{k}\right\|^{2} .
$$

As explained previously, an exact implementation of such a minimization is not possible: the functions $g^{i}$ are not known and exact calculation of inner products and other elementary operations is difficult. A possible solution is to apply the general method exposed in section 3.2, i.e. to work in a subspace $\mathcal{A}$. In this context, as demonstrated in [21] (chapter 6), it appears that functional PCA can be implemented by performing a classical PCA on the transformed coordinates (the $\beta\left(\tilde{g}^{i}\right)=U \alpha\left(\tilde{g}^{i}\right)$ vectors in $\mathbb{R}^{q}$, see 3.2 .2$)$ of the studied functions.

This method produces principal vectors in $\mathbb{R}^{q}$ that can be transformed back into principal functions in $\mathcal{A}$. For instance if $\tau$ is such a vector and $U$ is the Choleski factor of $\Phi$ defined in 3.2.2, then $U^{-1} \tau$ gives the coordinates of the corresponding principal function $\xi$ on the chosen basis for $\mathcal{A}$. Coordinates of the original functions on the principal function basis are obtained through inner products in $\mathcal{A}$. In practice they are obtained by canonical scalar products in $\mathbb{R}^{q}$ between $\beta\left(\tilde{g}^{i}\right)$ and $\tau$. Note that unlike conventional PCA, functional PCA usually works on centered, but not reduced to unit variance, data, because functional data must be seen as a unique entity rather than a set of unrelated coordinates with individual scales.

\subsection{Functional transformation}

A very interesting aspect of FDA is the possibility to implement a functional transformation before the data analysis phase. We will not cover in this paper registering techniques that allow to get rid of time shifting and other problems that can be interpreted as noise or distortion in the measurement process, i.e. problems related to the $x_{j}^{i}$. We refer the reader to [21] chapter five for an introduction on this complex topic.

We focus here on simpler functional transformations that provide different views of the same data. For instance, it is quite common in FDA to focus on the shape of the functions rather than on the actual values. A simple way to do this is to center and scale functions on a functional point of view, that is function by function. More precisely, we center $g$ by replacing it by $g_{c}$ defined as:

$$
g_{c}(x)=g(x)-\frac{1}{|V|} \int_{V} g(x) \mathrm{d} x .
$$


In this equation, $|V|$ is the volume of the compact $V$ (i.e. $|V|=\int_{V} \mathrm{~d} x$ ). The centered function is then scaled into $g_{s}$ defined as:

$$
g_{s}(x)=\frac{g_{c}(x)}{\frac{1}{|V|}\left\|g_{c}\right\|}
$$

An interesting aspect of those transformations is that they are based on elementary operations in the considered functional space. Therefore, they can be implemented using the coefficients that represent the input functions on the chosen projection space, as explained in section 3.2.2.

Another way to focus on shapes rather than on values is to calculate derivatives of the considered functions. To do so, we have to choose a projection space with a basis formed by derivable functions. Then, if $\tilde{g}^{i}=\sum_{k=1}^{q} \alpha_{k}\left(\tilde{g}^{i}\right) \phi_{k}$, obviously $\tilde{g}^{i(s)}=\sum_{k=1}^{q} \alpha_{k}\left(\tilde{g}^{i}\right) \phi_{k}^{(s)}$, where $f^{(s)}$ corresponds to the $s$-th derivative of $f$. It is therefore possible to work in $\mathcal{A}^{(s)}=\operatorname{span}\left(\phi_{1}^{(s)}, \ldots, \phi_{q}^{(s)}\right)$ exactly as we did in $\mathcal{A}$ (note that $\left(\phi_{1}^{(s)}, \ldots, \phi_{q}^{(s)}\right)$ might not be a free system anymore).

The special case of B-spline bases is very interesting. Indeed, it is clear that the derivative of a spline from $S_{\pi}^{\nu}$ is a spline of $S_{\pi}^{\nu-1}$; therefore it uses the same knots. Moreover, the coordinates of the derivative spline on the order $\nu-1 \mathrm{~B}$-spline basis can be calculated exactly and very easily with a finite difference equation, using the coordinates of the original spline on the order $\nu$ B-spline basis (see [10]). This allows to work with derivatives exactly as with the original functions.

\section{Simulation results}

The functional approach to Radial-Basis Function Networks and Multi-Layer Perceptrons is illustrated on spectrometric data coming from the food industry. This benchmark has been chosen here for illustration purposes: it permits to show which kind of functional (pre)processing is expected to give results similar to those on the original data, and which ones could lead to improved results. The models (RBFN and MLP) are optimized as detailed below. Nevertheless, their learning algorithm is chosen a priori, and no attempt is made to improve the results by comparing to other learning strategies; only comparisons between the possible ways to handle the functional data are discussed here. 


\subsection{Tecator spectra benchmark}

The Tecator data set [1] consists of 215 near-infrared absorbance spectra of meat samples, recorded on a Tecator Infratec Food and Feed Analyzer. Each observation consists in a 100-channel absorbance spectrum in the 850-1050 nm wavelength range. Each spectrum in the database is associated to a content description of the meat sample, obtained by analytic chemistry; the percentage of fat, water and protein are reported. The goal of the benchmark here is to predict the fat percentage from the spectra; this percentage is in the $[0.9,49.1]$ range.

From the 215 100-dimensional spectra, 43 are kept aside as a test set; the test set will not be used neither for model learning, nor for cross-validation (selection of the number of splines, of the number of PCA components, of the number of parameters in the models, etc.). The 172 remaining samples are used for model learning and validation, as detailed below.

It should be mentioned that the spectra are finely sampled, leading to very smooth curves; some of them are illustrated in Figure 1(a). It is therefore not expected that a functional preprocessing of the rough data, such as a spline decomposition, will lead to improved results, except if some a priori information is added, or if irregular sampling is artificially created by omitting data. This last point will be detailed in section 5 .

\subsection{Preprocessing the Tecator spectra}

In addition to working with rough spectra (100-dimensional measured vectors), three types of preprocessing are considered in the experiments.

First of all, a standard Principal Component Analysis (PCA) is performed. Unsurprisingly because of the smooth character of spectra, most of the information in terms of percentage of variance is contained in a few PCA components; this is illustrated in Figure 2 that shows the percentage of variance of the original data associated to each eigenvalue. As usually, data have been centered and reduced before applying the PCA; this induces a scaling that may have influence on the inner products and distance computations. The PCA is a non-functional preprocessing, as the continuous structure of spectra is not taken into account. It can be seen easily that the first principal component almost exactly represents the spectrum means, as illustrated by figure 3 .

To take into account the smooth character of spectra, a functional preprocessing is performed using bases of splines. Splines of degrees 3, 4 and 5 (respectively order 4,5 and 6 ) are used; the two last ones are aimed to be derived 

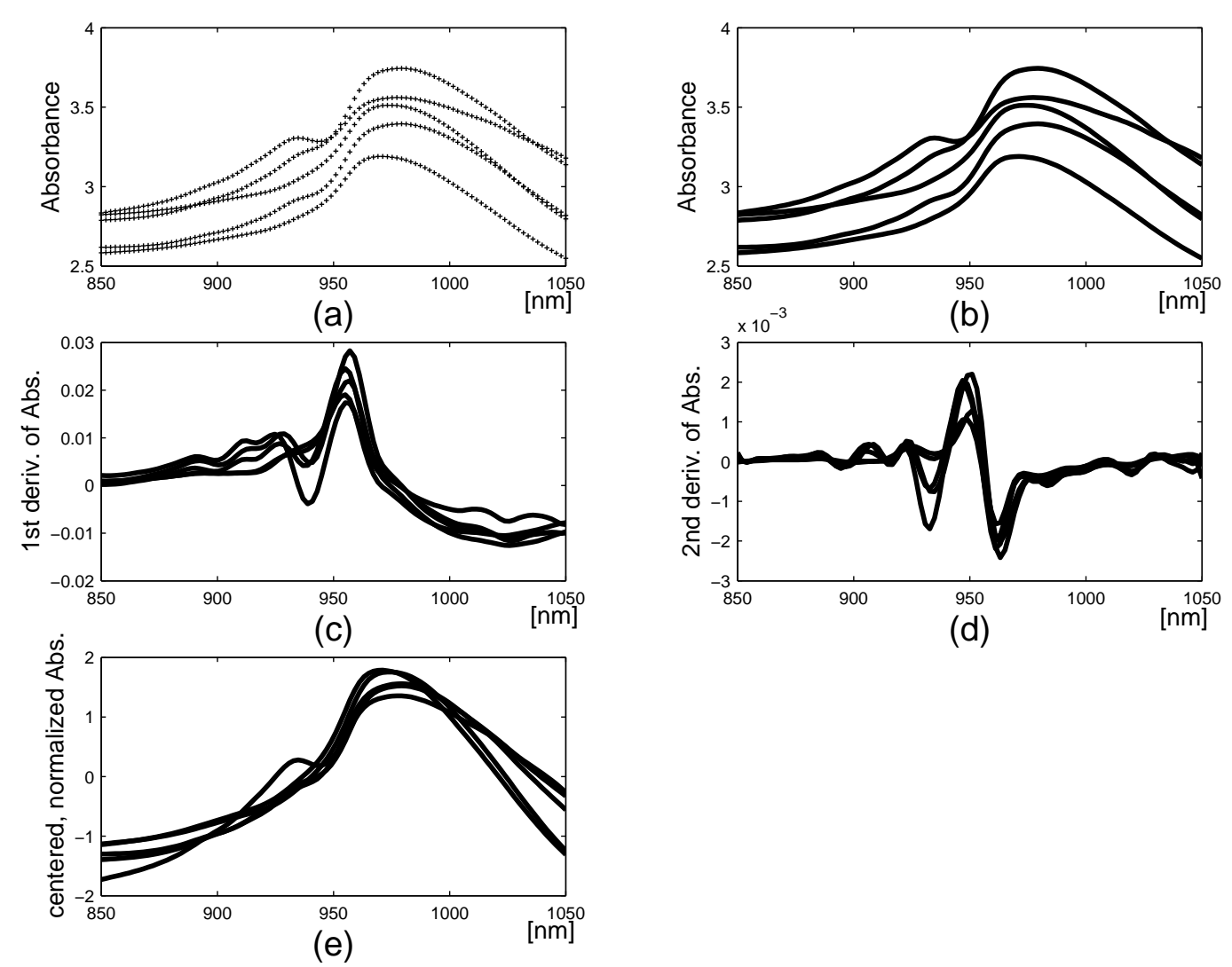

(d)

Figure 1. Five spectra from the Tecator benchmark; (a) original samples, (b) 4th-order spline approximation, (c) derivative of 5th-order spline approximation, (d) second derivative of 6th-order spline approximation, (e) 4th-order spline approximation after functional centering and reduction

(respectively once and twice) in order to work with derivatives of spectra rather than with the original ones.

The numbers of basis functions selected by the leave-one-out procedure detailed in section 3.3.2 are respectively 48, 43 and 32 for the 4 th-, 5th- and 6th-order splines. Figures 1(b), (c) and (d) respectively show the spectra approximated by 4th-order spline, the derivative of the 5th-order spline approximation, and the second derivative of the 6th-order spline approximation.

A last preprocessing takes into account a priori information on the spectra. As spectrometry experts know that the shape of the spectra is by far more important than their mean value (for the fat prediction problem), centering and reducing them (to unit variance) avoids that their average could influence the models. Figure 1(e) shows the results of this functional centering and reduction (performed on the 4 th-order spline approximation). 


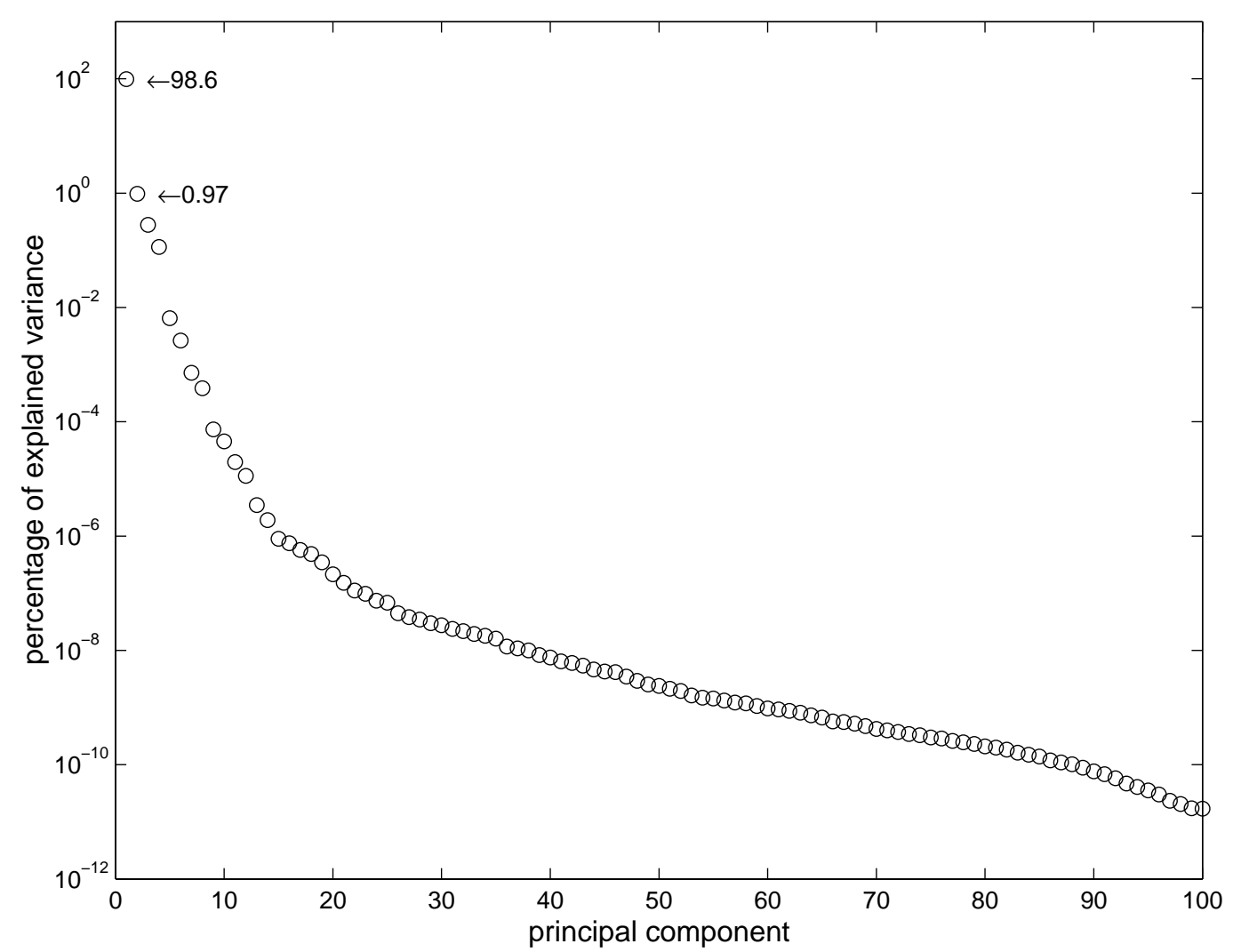

Figure 2. Percentage of variance explained by each of the PCA principal components

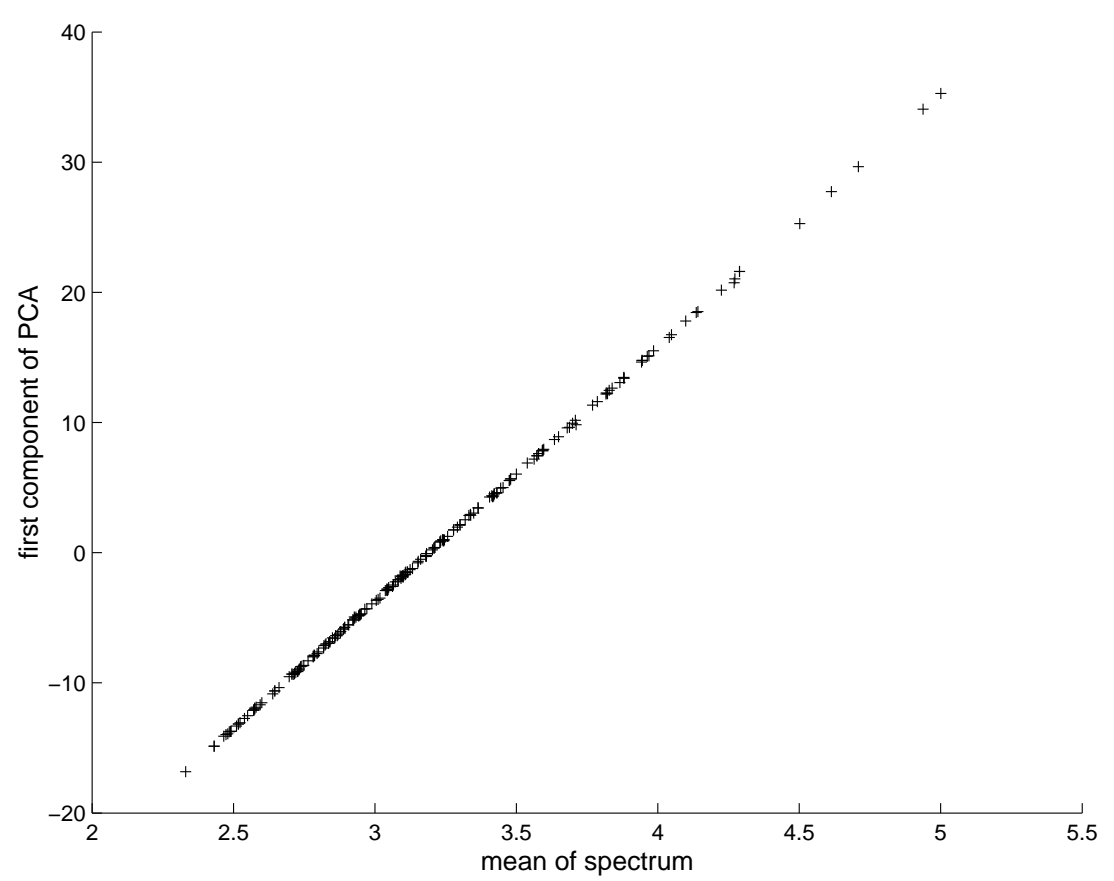

Figure 3. Coordinates on the first principal component as a function of the spectrum mean 


\subsection{Using the Radial-Basis Function Network model}

A number of experiments have been conducted on the Tecator benchmark, with the Radial-Basis Function Network model described in section 2.3. The model parameters are learned through an OLS procedure, as detailed in [6]. In short, the OLS procedure is a forward selection algorithm that incrementally chooses centers (and associated Gaussian functions) among a set of candidates; here the candidates are the data in the learning set. The error criterion used for the selection is the sum of the squared errors made by the model and a regularization term [19]; the contribution of each candidate to the error criterion is measured, and the one that minimizes this contribution is chosen. The incremental procedure is performed up to a high number of selected Gaussian functions (100 in the following experiments). Next, a 4-fold crossvalidation procedure is used on the learning set (172 spectra) to select the optimal number of Gaussian functions, according to the sum-of-squares error criterion.

Ten experiments are performed:

(1) The 100-dimensional rough spectra are used as inputs to the RBFN.

(2) The spectra are preprocessed by PCA, and the first 20 PCA components are kept (100\% of the original variance is preserved, as shown in Figure 2).

(3) The same PCA preprocessing is used, but now the number of PCA components that are kept is selected according to a 4-fold cross-validation procedure on the learning set; this optimization leads to a choice of 5 PCA components.

(4) The PCA components are used, but they are whitened (centered and scaled to unit variance); unlike the functional centering and reduction detailed in the previous section, the whitening here is a conventional one, i.e. it is applied component by component. The purpose of this whitening is to allow each component to have the same importance. Without this whitening, the first component would have much more importance than the other ones, while its influence on the fat prediction problem is known to be low. The number of PCA components that are kept is selected by 4-fold cross-validation as above: 6 components are selected.

(5) The 48 coefficients of the 48 th-order splines are used as inputs to the RBFN.

(6) A functional PCA (see Section 3.4) is performed on the 4th-order splines with 48 coefficients; 20 coefficients are kept.

(7) A functional PCA is performed on the 4th-order splines with 48 coefficients, and the PCA coefficients are whitened; A 4-fold cross-validation selects 6 components.

(8) A functional centering and reduction is applied to the 4th-order spline 
approximation; the 48 resulting coefficients are used as inputs to the RBFN.

(9) The 5th-order spline is derived, and the 42 resulting coefficients are used as inputs to the RBFN.

(10) The 6th-order spline is derived twice, and the 30 resulting coefficients are used as inputs to the RBFN.

Table 1 shows the results of these ten experiments. All results are given in terms of Root Mean Square Error (RMSE) on the test set.

\begin{tabular}{|c|c|c|}
\hline Experiment \# & Experiment & Result on test set \\
\hline 1 & 100-dimensional original data & 4.97 \\
\hline 2 & PCA, no whitening, 20 components & 4.99 \\
\hline 3 & $\begin{array}{l}\text { PCA, no whitening, a } 4 \text {-fold cross- } \\
\text { validation selects the } 5 \text { first compo- } \\
\text { nents }\end{array}$ & 4.85 \\
\hline 4 & $\begin{array}{l}\text { PCA, whitening, a } 4 \text {-fold cross- } \\
\text { validation selects the } 5 \text { first compo- } \\
\text { nents }\end{array}$ & 1.94 \\
\hline 5 & $\begin{array}{l}\text { 4th-order B-splines, } 48 \text { coefficients, } \\
\text { no whitening }\end{array}$ & 4.59 \\
\hline 6 & $\begin{array}{l}\text { 4th-order B-splines, } 48 \text { coefficients, } \\
\text { functional PCA, no whitening, } 20 \\
\text { components }\end{array}$ & 4.59 \\
\hline 7 & $\begin{array}{l}\text { 4th-order B-splines, } 48 \text { coefficients, } \\
\text { functional PCA, whitening, a } 4 \text {-fold } \\
\text { cross-validation selects the } 6 \text { first } \\
\text { components }\end{array}$ & 1.83 \\
\hline 8 & $\begin{array}{l}\text { 4th-order B-splines after functional } \\
\text { centering and reduction, } 48 \text { coeffi- } \\
\text { cients, no whitening }\end{array}$ & 1.64 \\
\hline 9 & $\begin{array}{l}\text { first derivative of } 5 \text { th-order B-splines, } \\
42 \text { coefficients, no whitening }\end{array}$ & 0.90 \\
\hline 10 & $\begin{array}{l}\text { second derivative of } 6 \text { th-order B- } \\
\text { splines, } 30 \text { coefficients, no whitening }\end{array}$ & 0.81 \\
\hline
\end{tabular}

Table 1

RMSE on the test set for the RFBN experiments (see text for details)

The following conclusions can be drawn.

- The results of experiments 1 and 5 are roughly the same. Indeed the decomposition into splines does not bring any improvement, as there is a nearly perfect correspondence between the original spectra and their spline ap- 
proximation. The use of the scaling after Cholesky decomposition of the $\Phi$ matrix (see section 3.2) guarantees that the results obtained after spline preprocessing will be similar to those on the original spectra, as the latter are very smooth (therefore almost perfectly approximated by splines).

- The non-functional PCA reduction (experiments 2 and 3) does not bring any improvement to the results; the reduction to unit variance included in the PCA does not seem to be advantageous here; actually the variances of the original data components are more or less identical in the data set, therefore the reduction has little effect.

- The centering and reduction of the PCA coefficients (experiment 4) improves the results; indeed the influence of the first PCA component is strongly decreased in this process. The first PCA component is proportional to the spectrum averages (see Figure 3), which are known to be of little influence in the fat prediction problem.

- Similarly to the fact that experiments 1 and 5 give approximately the same results (the decomposition into splines does not bring much additional smoothness as the original spectra are already very smooth), experiments 2 and 6 on one side, and experiments 4 and 7 on the other side, lead to similar results. In experiments 2 and 4 an initial reduction to unit variance is performed and not in experiments 6 and 7 , but as mentioned above this reduction does not bring any improvement.

- The functional centering and reduction (experiment 8) also improves the predictions compared to the original ones. The improvement also results from the removal of the spectrum averages, and is comparable to the centering and reduction of the PCA components.

- As expected, taking the first and second derivatives of the spectra (more precisely, taking the first and second derivative of their 5th- and 6th- order spline approximations respectively) focuses on the differences in the spectra shapes, therefore allowing a better prediction of fat content.

\subsection{Using the Functional Multi-layer Perceptron model}

The original contributor of the Tecator data set used traditional MLP together with PCA to build a regression model [5, 30]. In [5] Borggaard and Thodberg use a standard MLP on the 10 first principal components. They use early stopping to avoid overfitting and report a RMSE of 0.65. In [30] Thodberg reports better results based on a more complex training algorithm and model: he uses a weight decay regularization term and chooses meta-parameters through a Bayesian approach. More precisely, he uses an one hidden layer perceptron with additional direct connections from the inputs to the output node (introducing this way a linear term) and three separate regularization terms, one for each layer and one for the weights of the linear term. The values of those weight decays as well as the number of hidden neurons are determined by a 
Bayesian estimation of the generalization error. Using the 10 first principal components Thodberg obtains a RMSE of 0.55 . In order to improve the results, he combines the 10 best MLPs obtained out of 40 trained MLPs in an ensemble model that reaches a RMSE of 0.52. Finally, he embeds into the Bayesian meta-parameters selection the determination of the optimal number of principal components. He selects this way 12 principal components for a RMSE of 0.42. Using a smoothed version of this input selection (based on two other weight decay parameters) he even managed to reach a RMSE of 0.36 with 13 principal components.

The goal of the proposed simulations is not to reproduce Thodberg's results but simply to illustrate the positive effects of the functional methodology. Therefore, a simplified neural model has been used in order to focus on the preprocessing. The chosen model is a single classical one hidden layer perceptron with no direct connection, together with a single regularization term (which is not used for bias terms). Meta-parameters (the weight decay, the number of hidden neurons, etc.) are chosen through the same 4-fold cross-validation procedure (as with the RBFN models). No ensemble model or smooth variable selection is used. Training itself is done by a second-order gradient descent method starting from 60 different initial random weight vectors (for each experiment). The best MLP obtained from those random weight is kept according to the sum-of-squares error criterion (on the training set) combined with the regularization term. The number of hidden neurons varies from 1 to 6 . In all experiments, the best 4 -fold cross-validation was obtained with 2 hidden units.

Five experiments were conducted:

(1) The spectra are preprocessed by PCA and the number of PCA components is selected by the 4 -fold cross-validation procedure on the learning set. This experiment plays the role of the reference one as we do not use the sophisticated method of Thodberg.

(2) The spectra are converted into their 4th-order B-splines representation, a functional PCA is conducted and the number of components to retain is again selected by the 4 -fold cross-validation procedure.

(3) We do the same as in the previous experiment but we apply a functional centering and reduction before the functional PCA.

(4) The 5th-order spline is derived; a functional PCA is conducted on the resulting functions and the number of components to retain is again selected by the 4 -fold cross-validation procedure.

(5) The 6th-order spline is derived twice; functional PCA is conducted on the resulting functions and the number of components to retain is again selected by the 4 -fold cross-validation procedure.

An important point is that PCA coordinates are always whitened before being 
used by the MLP. Indeed as the explained variance is very much concentrated in the first coordinate, the variation range is quite different from the different inputs of the MLP. This means that the corresponding weights should be very different: this is not compatible with the weight decay regularization as large weights (corresponding to large range) are more heavily penalized than small weights.

Another difference with the RBFN experiments is that a PCA (functional or classical) is always done before submitting the data to the MLP. Our goal was to avoid huge training times as well as high dimensionality related problems induced by the size of the data. The experiments have been limited this way to at most 18 principal components to use a reasonable input size for the MLP.

Table 2 summarizes the results of those five experiments. All results are given in terms of Root Mean Square Error (RMSE) on the test set.

\begin{tabular}{|c|l|c|}
\hline Experiment \# & Experiment & Result on test set \\
\hline 1 & $\begin{array}{l}\text { PCA, a 4-fold cross-validation selects } \\
\text { the 12 first components, whitening }\end{array}$ & 0.49 \\
2 & $\begin{array}{l}\text { 4th-order B-splines, 48 coefficients, } \\
\text { functional PCA, a 4-fold cross- } \\
\text { validation selects the 12 first compo- } \\
\text { nents, whitening }\end{array}$ & 0.49 \\
3 & $\begin{array}{l}\text { 4th-order B-splines, 48 coefficients, } \\
\text { functional PCA on centered and } \\
\text { reduced functions, 4-fold cross- } \\
\text { validation selects the 11 first compo- } \\
\text { nents, whitening } \\
\text { first derivative of 5th-order B-splines, } \\
42 \text { coefficients, functional PCA, a 4- } \\
\text { fold cross-validation selects the 15 } \\
\text { first components, whitening } \\
\text { second derivative of 6th-order B- } \\
\text { splines, 30 coefficients, functional } \\
\text { PCA, 4-fold cross-validation selects } \\
\text { the 13 first components, whitening }\end{array}$ & 0.44 \\
\hline
\end{tabular}

Table 2

RMSE on the test set for the MLP experiments

These results justify the following comments.

- The reference experiment (number 1) shows that the chosen experimental setting is comparable to the one used by Thodberg. Indeed, the obtained MLP performs slightly better than the one selected by Thodberg ( 0.49 versus 0.55$)$ probably because we choose automatically the appropriate num- 
ber of principal components. On the other hand, our simpler setting cannot reach the best performances reported in [30] probably because the regularization method is less flexible than the one used by Thodberg.

- The best functional preprocessing allows to improve slightly the test performances (from 0.49 to 0.44 , that is about $10 \%$ ) in a rather simple way. Moreover, a higher-level 4-fold cross-validation in which the method itself is automatically chosen in addition to the number of hidden neurons, of principal components, etc., chooses the model produced by experiment number 3, i.e. the functional preprocessing that leads to the best test performances.

- MLP performances are better than RBFN ones, but the price to pay in terms of calculation time is huge. A full experiment with MLPs takes about 200 times more computational time than a similar experiment with a RBFN network. We face here one of the classical tradeoffs between model design time and model accuracy. As the functional approach introduces additional possibilities such as functional preprocessing (derivative, centering, etc.), it makes the training problem even more important. Exploring all the available functional preprocessing solutions can become nearly impossible for MLP models while remaining feasible for RBFN networks. Moreover, it appears clearly that $\mathrm{RBFN}$ results cannot be used as a guideline for the construction of a good MLP model. Indeed, derivatives were really useful for improving the RBFN results, whereas they give worse performances in the case of the MLP.

\section{$5 \quad$ Missing data}

\subsection{A semi-artificial benchmark}

A nice property of FDA is its ability to deal with irregular sampling. In some situations, it happens that the sampling process has some variation between input functions. This is the case for instance in medical time series where patients decide on their own when to be monitored by doctors. Irregular sampling appears also for gesture recognition like cursive handwriting recognition for personal digital assistant: while the sampling rate is fixed, gestures have different execution times that depend on the context of execution rather than on the gesture performed. Therefore, some registration is needed; its effect is to transform the regular sampling into a gesture-specific one.

The goal of this section is to illustrate the way FDA solves irregular sampling problems in its simplest form: a regular sampling with missing data. To do so, a semi-artificial data set was created by removing data from the Tecator data set used in the previous section. More precisely, $10 \%$ of the observations in each spectrum of the data set were removed at random (therefore 90 absorbances 
out of 100 are kept). Of course, spectrometers provide regular spectra and the obtained data are not representative of spectrometric problems. The goal is simply here to illustrate the possibilities of FDA with data for which we have reference performances.

\subsection{Functional preprocessing}

The function representation strategy described in section 3 applies to arbitrary sampling. Therefore the procedure followed in section 4.2 does not have to be modified: the coordinates of the considered functions on B-spline bases of various orders are calculated. The leave-one-out procedure selects less Bsplines than with complete data. Indeed, the number of basis functions are respectively 28, 27 and 21 for the 4th-, 5th- and 6th-order splines. This reduction is easily explained by the fact that B-splines are localized functions. When the number of knots is high, the support of individual B-splines is small and it can happen that for a given spectrum no observation is available on the whole support of a B-spline. In this case, the corresponding coordinate cannot be calculated. Before this extreme situation, coefficients become numerically unstable because some B-spline supports do not contain enough observations to allow a correct estimation of the corresponding coefficients.

\subsection{Results}

The Tecator benchmark with $10 \%$ of missing data as described above was used for experiments with a RBFN network. Only the most interesting methods from section 4.3 were used on these data, namely experiments number 9 and 10, on the first and second derivative of the 5th- and 6th-order splines respectively. Table 3 gives the results of the two experiments (to simplify the comparison with section 4.3, the same experiment numbers are kept). It clearly appears that the functional approach solves the problem of missing data in this particular situation.

\begin{tabular}{|c|l|c|}
\hline Experiment \# & Experiment & Result on test set \\
\hline 9 & $\begin{array}{l}\text { first derivative of the 5th-order B- } \\
\text { splines, 26 coefficients, no whitening }\end{array}$ & 0.05 \\
10 & $\begin{array}{l}\text { second derivative of the 6th-order B- } \\
\text { splines, 19 coefficients, no whitening }\end{array}$ & 0.80 \\
\hline
\end{tabular}

Table 3

RMSE on the test set for the RBF experiments with missing data

As for the RBFN, experiments were limited to the best preprocessing methods for use with the MLP, namely raw functional data followed by a func- 
tional PCA as well as centered and reduced functional data also followed by a functional PCA. All meta-parameters (including the number of principal components) were selected by a 4 -fold cross-validation, exactly as in section 4.4. Table 4 gives the results of the two experiments. It also clearly appears that the functional approach solves here the problem of missing data.

\begin{tabular}{|c|l|c|}
\hline Experiment \# & Experiment & Result on test set \\
\hline 2 & $\begin{array}{l}\text { 4th-order B-splines, 28 coefficients, } \\
\text { functional PCA, a 4-fold cross- } \\
\text { validation selects the 12 first compo- } \\
\text { nents, whitening } \\
3\end{array}$ & $\begin{array}{l}\text { 4th-order B-splines, 28 coefficients, } \\
\text { functional PCA on centered and } \\
\text { reduced functions, a 4-fold cross- } \\
\text { validation selects the 11 first compo- } \\
\text { nents, whitening }\end{array}$ \\
\hline
\end{tabular}

Table 4

RMSE on the test set for the MLP experiments with missing data

\subsection{Alternative solutions}

The standard way of dealing with missing data is to use an imputation method that will reconstruct the needed values. The simplest imputation method consist in replacing a missing value by the mean of available values for the corresponding variable.

A more interesting method consists in using a $k$-nearest neighbors approach: given an input with missing values, its $k$-nearest neighbors, among inputs that do not miss the corresponding value, are calculated and the missing value is replaced by the mean of this variable for the $k$-nearest neighbors. Of course the distance has to be adapted to take care of the missing data problem. A possible solution is simply to discard missing values. Let us denote $n m(x)$ the set of indices $j$ for which $x_{j}$ is not missing. Then the distance used for the nearest neighbor $y$ calculation is:

$$
d(x, y)=\frac{1}{|n m(x) \cap n m(y)|} \sum_{j \in n m(x) \cap n m(y)}\left(x_{j}-y_{j}\right)^{2},
$$

where $|A|$ is the cardinal of the set $A$.

When imputation has been done, a standard processing method can be applied. Section 4.3 showed that non-functional approaches give very bad results for the RBF network; therefore it has been decided to study the imputation 
method associated to a standard processing approach only for the MLP model. Two experiments have been conducted in this way:

(1) Missing values are imputed using the mean approach to reconstruct spectra in $\mathbb{R}^{100}$; then a standard PCA is applied. The number of principal components to retain is determined by the 4 -fold cross-validation used for other meta-parameters optimization.

(2) Missing values are imputed using the $k$-nearest neighbors approach. Resulting spectra are processed by a regular PCA. Both $k$ and the number of principal components are determined by 4 -fold cross-validation.

Table 5 summarizes the obtained results, which are quite bad, especially for the mean approach. It appears in fact that the reconstruction is very bad because of the mean spectrum effect already encountered with the RBFN model. Indeed, spectra with similar shape but very different means can correspond to similar values of fat. Unfortunately, this means that reconstructing the shape of the spectra without using an expert knowledge is very difficult.

\begin{tabular}{|c|l|c|}
\hline Experiment \# & Experiment & Result on test set \\
\hline 1 & $\begin{array}{l}\text { mean imputation, PCA, a 4-fold } \\
\text { cross-validation selects the 5 first } \\
\text { components, whitening }\end{array}$ & 7.13 \\
2 & $\begin{array}{l}\text { 4-nearest neighbors imputation, } \\
\text { PCA, a 4-fold cross-validation } \\
\text { selects the 12 first components, } \\
\text { whitening }\end{array}$ & 1.87 \\
\hline
\end{tabular}

Table 5

RMSE on the test set for the MLP experiments with missing data and classical imputation methods

A possibility to take into account this kind of expert knowledge into the imputation process without relying on a functional approach is to center and scale each spectrum before applying the imputation method. More precisely, in a way modeled after the functional scaling described in section $3.5, x$ is replaced by $x^{s}$ defined by:

$$
x_{i}^{s}=\frac{x_{i}-\sum_{j \in n m(x)} x_{j}}{\sqrt{\sum_{j \in n m(x)}\left(x_{j}-\sum_{k \in n m(x)} x_{k}\right)^{2}}}
$$

The same experiments as described above were conducted with this additional preprocessing phase. Table 6 summarizes the obtained results, which are much better than without the inclusion of the expert knowledge. Even so, results are still worse than the ones obtained by the functional preprocessing. Moreover, the expert knowledge corresponds clearly to a functional point of view and the imputation methods based on it should be considered as almost functional 
methods.

\begin{tabular}{|c|l|c|}
\hline Experiment \# & Experiment & Result on test set \\
\hline 1 & $\begin{array}{l}\text { expert pre-processing, mean imputa- } \\
\text { tion, PCA, a 4-fold cross-validation } \\
\text { selects the 9 first components, } \\
\text { whitening }\end{array}$ & 1.82 \\
2 & $\begin{array}{l}\text { expert pre-processing, 8-nearest } \\
\text { neighbors imputation, PCA, a 4-fold } \\
\text { cross-validation selects the 9 first } \\
\text { components, whitening }\end{array}$ & 0.85 \\
\hline
\end{tabular}

Table 6

RMSE on the test set for the MLP experiments with missing data and expert imputation methods

\section{Conclusion}

Functional Data Analysis (FDA) is an extension of traditional data analysis to functional data, lying in an infinitely-dimensional space. Examples of functional data are spectra, temporal series, spatio-temporal images, gesture recognition data such as cursive handwriting patterns, etc. Functional data are rarely known in practice; instead lists of input-output pairs (one for each functional data) are usually known. Their sampling can be irregular, even different from one functional data to another.

This paper shows how to extend the Radial-Basis Function Network (RBFN) and Multi-Layer Perceptron (MLP) models to functional data inputs. A particular emphasis is put on how to handle functional data in practical situations, i.e. when they are known through list of sampled values. In particular, various possibilities for functional processing are presented, including the projection on smooth bases, Functional Principal Component Analysis (FPCA), functional centering and reduction and the use of differential operators. It is shown how to incorporate these functional preprocessings into the RBFN and MLP models, and how to take into account the non-orthogonality of basis vectors in the case of preprocessing by projection.

The methods are applied to a benchmark in spectroscopy. The advantages and limitations of the various FDA approaches are discussed on this benchmark, both in the RBFN and MLP cases. It is shown how an adequately chosen functional preprocessing can improve the way functional data are handled into data analysis methods.

The case of irregularly-sampled functional data is discussed through the same 
benchmark where a percentage of values have been artificially removed. It is shown that the FDA approach is robust to such missing data, while traditional imputation techniques fail to provide adequate results.

The FDA approach, combined with an appropriate choice of how to represent the functional data, may reveal interesting in a variety of situations where the smooth character or the irregular sampling of data has to be taken into account.

\section{References}

[1] Tecator dataset. Available on statlib: http://lib.stat.cmu.edu/datasets/tecator.

[2] Christophe Abraham, Pierre-André Cornillon, Eric Matzner-Lober, and Nicolas Molinari. Unsupervised curve clustering using b-splines. Scandinavian Journal of Statistics, 30(3):581-595, September 2003.

[3] Philippe Besse, Hervé Cardot, and Frédéric Ferraty. Simultaneous nonparametric regressions of unbalanced longitudinal data. Computational Statistics and Data Analysis, 24:255-270, 1997.

[4] Philippe Besse and Jim Ramsay. Principal component analysis of sampled curves. Psychometrica, 51:285-311, 1986.

[5] C. Borggaard and H.H. Thodberg. Optimal minimal neural interpretation of spectra. Analytical Chemistry, 64:545-551, 1992.

[6] S. Chen, F.N. Cowan, and P. M. Grant. Orthogonal least squares learning algorithm for radial basis function networks. IEEE Trans. on Neural Networks, 2(2):302-309, 1991.

[7] Brieuc Conan-Guez and Fabrice Rossi. Multilayer perceptrons for functional data analysis: a projection based approach. In José R. Dorronsoro, editor, Artificial Neural Networks - ICANN 2002, pages 667-672, Madrid, August 2002. Springer.

[8] J. Dauxois and A. Pousse. Les analyses factorielles en calcul des probabilités et en statistiques : essai d'étude synthétique. Thèse d'état, Université Paul Sabatier, Toulouse, 1976.

[9] J. Dauxois, A. Pousse, and Y. Romain. Asymptotic theory for the principal component analysis of a vector of random function: some applications to statistical inference. Journal of Multivariate Analysis, 12:136-154, 1982.

[10] Carl de Boor. Fundamental Developments of Computer-Aided Geometric Modeling (Les Piegl, editor), chapter B-spline basics, pages 27-49. Academic Press, London, 1993.

[11] Nicolas Delannay, Fabrice Rossi, Brieuc Conan-Guez, and Michel Verleysen. Functional radial basis function network. In Proceedings of ESANN 2004, pages 313-318, Bruges, Belgium, April 2004. 
[12] T. Evgeniou, M. Pontil, and T. Poggio. Regularization networks and support vector machines. Advances in Computational Mathematics, 13(1):150, 2000.

[13] Frédéric Ferraty and Philippe Vieu. The functional nonparametric model and application to spectrometric data. Computational Statistics, 17(4), 2002.

[14] Frédéric Ferraty and Philippe Vieu. Curves discriminations: a nonparametric functional approach. Computational Statistics and Data Analysis, 44(1-2):161-173, 2003.

[15] Louis Ferré and Anne-Françoise Yao. Functional sliced inverse regression analysis. Statistics, 37(6):475-488, November/December 2003.

[16] F. Girosi, M. Jones, and T. Poggio. Regularization theory and neural networks architectures. Neural Computation, 7(2):219-269, 1995.

[17] Gareth M. James and Catherine A. Sugar. Clustering for sparsely sampled functional data. Journal of American Statistical Association, 98:397-408, 2003.

[18] Teuvo Kohonen. Self-Organizing Maps. Springer Verlag, New York, 1997.

[19] Mark J. L. Orr. Regularization in the selection of radial basis function centers. Neural Computation, 7(3):606-623, 1995.

[20] J. Park and I. W. Sandberg. Universal approximation using radial basis function networks. Neural Computation, 3(2):246-257, 1991.

[21] Jim Ramsay and Bernard Silverman. Functional Data Analysis. Springer Series in Statistics. Springer Verlag, June 1997.

[22] Fabrice Rossi and Brieuc Conan-Guez. Functional multi-layer perceptron: a nonlinear tool for functional data analysis. Neural Network, 2004. To be published.

[23] Fabrice Rossi and Brieuc Conan-Guez. Functional preprocessing for multilayer perceptrons. In Proceedings of ESANN 2004, pages 319-324, Bruges, Belgium, April 2004.

[24] Fabrice Rossi, Brieuc Conan-Guez, and Aïcha El Golli. Clustering functional data with the som algorithm. In Proceedings of ESANN 2004, pages 305-312, Bruges, Belgium, April 2004.

[25] Fabrice Rossi, Brieuc Conan-Guez, and François Fleuret. Functional data analysis with multilayer perceptrons. In Proceedings of IJCNN 2002 (WCCI 2002), volume 3, pages 2843-2848, Honolulu, Hawai, USA, May 2002. IEEE/NNS/INNS.

[26] Fabrice Rossi, Brieuc Conan-Guez, and François Fleuret. Theoretical properties of functional multilayer perceptrons. In Proceedings of ESANN 2002, pages 7-12, Bruges, Belgium, April 2002.

[27] Irwin W. Sandberg. Notes on weighted norms and network approximation of functionals. IEEE Transactions on Circuits and Systems-I: Fundamental Theory and Applications, 43(7):600-601, July 1996.

[28] Irwin W. Sandberg and Lilian Xu. Network approximation of inputoutput maps and functionals. Circuits Systems Signal Processing, 15(6):711-725, 1996. 
[29] Maxwell B. Stinchcombe. Neural network approximation of continuous functionals and continuous functions on compactifications. Neural Networks, 12(3):467-477, 1999.

[30] Hans Henrik Thodberg. A review of Bayesian neural networks with an application to near infrared spectroscopy. IEEE Trans. on Neural Networks, 7(1):56-72, 1996.

[31] V. Vapnik. Statistical Learning Theory. Wiley, New York, 1998. 